\title{
Sequential and Tonal Markedness in Zijin Hakka Tone Sandhi Jingyi Chen
}

\author{
Sichuan International Studies University, Chongqing ,404100, China \\ delijingyic@gmail.com
}

\begin{abstract}
This paper examines two complex tone sandhi patterns in Zijin Hakka by using rule-based generative model, and Optimality Theory. The first sandhi pattern involves both assimilation and dissimilation processes. Assimilation forces adjacent tone contour features to agree, while dissimilation forces adjacent elements to be different at the contour level. These tonal alternations are captured by constraints OCP-C(Ih), IDENT-IO-t-L, *peak pitch and Align last contour right. The second sandhi rule has neutralization process. When the rising tones followed by falling tone in Zijin Hakka, the whole two adjacent syllables will neutralize to 13-351. This fact is captured by constraint ${ }^{*}$ Ih.hl, F, IDENT-IO-t-L, * peak pitch, Align last contour right and F(\#Ih.).
\end{abstract}

Keywords: Hakka, tone sandhi, rule-based model, Bao's tonal geometry model, Optimality Theory.

\section{Introduction}

Tone sandhi is the tonal alternation between adjacent tones. The most common motivation of tone sandhi are assimilation and dissimilation processes. As assimilation means that the alternation of tone results in a similarity between adjacent tones, on the contrary, dissimilation results in alternation of tone that makes adjacent tones less like each other. Assimilation has been found in several Chinese dialects including Cantonese and Chaozhou and especially the neutral tone sandhi in Mandarin. (Lin, 2011) And dissimilation is also found in Mandarin tonal alternation of third tone sandhi. In Huishan Lin's study towards Taiwan Dongshi Hakka, the function of assimilation and dissimilation in tonal sandhi is evaluated. Other previous study also analyzed the effect of assimilation and dissimilation.

The markedness status of tones is another critical reason for tone sandhi. As previous study found that in many languages, different positions have preference of tone (Lacy 1999,2002), Lin (2011) also examined the preference of tones in different position in Dongshi Hakka.

The tone sandhi in Zijin Hakka is unique as it has two specific tonal alternations. One of which involves the effect of both assimilation and dissimilation in its tonal change in which the assimilatory requires intersyllabic tone feature to agree, and the dissimilatory prohibits the combination of rising contour tones. Another tone sandhi involves neutralization that four different tone combinations change to have the same feature. The interesting thing is that this tone sandhi will occur only when the beginning tone is rising tone $(13,15)$ which indicates a preference of tone on the position after rising tone. In an OT framework, this paper demonstrates markedness constraints to account for assimilation, dissimilation and neutralization as well as preference of tone happen in Zijin Hakka.

The rest of paper is organized as follows. In section 2, a brief factual description of the basic fact of Zijin Hakka tone is given and the tone sandhi is presented using rule-based models. And then the OT analysis to explain the first tone sandhi that has both assimilation and dissimilation is included in section 3. In section 4, the second tone sandhi of neutralization is examined and analyzed through OT constraint. The last part offers the conclusion to all these analyses.

\section{Zijin Hakka Tonal Facts and Previous OT Analyses}

Zijin Hakka is a regional branch of Hakka spoken by people live in Zijin county, Heyuan City, Guangdong Province in south China. As there are counties with Hakka speakers as the majority, so some of these Hakka dialects are not mutually ineligible with each other. The common Hakka dialects is Meizhou Hakka, Huizhou Hakka, Heyuan Hakka, which is mainly spoken in Guangdong province, Changting Hakka, which is the western Fujian dialect, and Dongshi Hakka spoken in Taiwan. Huishan Lin (2010) presents the tone sandhi in Dongshi Hakka, and Matthew Y Chen (2004) analyzes Changting Hakka sandhi; however, Zijin Hakka has some specific sandhi that has not be reported yet. 


\subsection{Tone Definition}

In this paper, a new tone is defined if at least one of its range of pitches, the way pitches changes, and the length of the tone is different with all other tones that have already ascertained.

Methodology to define tone

List all words of Zijin Hakka that has been found in a "dictionary".

Makes informant pronounce a word (x) randomly selected from dictionary.

Makes informant pronounce a word (y) randomly selected from an existed tone group:

Compare the tone of $\mathrm{x}$ and $\mathrm{y}$, if they are identical, group $\mathrm{x}$ together with $\mathrm{y}$.

If tone of $\mathrm{x}$ and $\mathrm{y}$ are different, select another word from an untested tone in existed tone group to be y, repeat step C.i.

If a new word doesn't belong to any existed tone groups, create a new tone group that contains the new word.

Repeat B to D until large number of words in dictionary are tested and grouped.

Randomly choose two tone ( $\mathrm{x}$ and $\mathrm{y}$ ) from dictionary, ask informant whether $\mathrm{x}$ and $\mathrm{y}$ are of same tone or not, verify their answer to previous grouping result. Repeat. Notice that tester will not get knowledge of how words are grouped.

As a result, words are separated into 7 groups:

Table 1. Zijin Hakka tones

\begin{tabular}{|c|c|}
\hline $\begin{array}{l}\text { Zijin } \\
\text { Hakka }\end{array}$ & \\
\hline 15 & 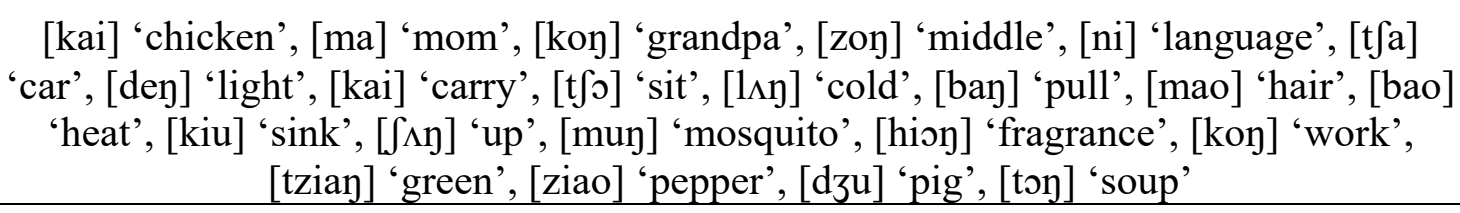 \\
\hline 44 & $\begin{array}{l}\text { [miao] 'cat', [kiao] 'cry', [tiao] 'jump', [liao] 'play', [siao] 'smile', [kian] 'piece', } \\
\text { [zui] 'guity', [yi] 'two', [yoi] 'outside', [qia] 'thank', [su] 'tree', [ka] 'marry', [zo] } \\
\text { 'do', [si] 'four', [Ju] 'column', [pui] 'boil', [kiu] 'old', [miay] 'life', [lu] 'road', [puy] } \\
\text { 'sprinkle' }\end{array}$ \\
\hline 11 & $\begin{array}{c}\text { [niuy] 'cow', [pa] 'climb', [na] 'teeth', [tsa] 'tea', [nin] 'people', [Jon] 'long', [lon] } \\
\text { 'dragon', [mun] 'door', [ten] 'sweet', [toy] 'sugur', [tai] 'big', [sic] 'small', [niay] } \\
\text { 'year', [wun] 'smell', [koi] 'cover', [lon] 'cool }\end{array}$ \\
\hline 31 & $\begin{array}{l}\text { [ko] 'permit', [hao] 'good', [ziu] 'walk', [fui] 'water', [mion] 'net', [lao] 'old', [mu] } \\
\text { 'five', [fo] 'fire', [kon] 'say', [tzao] 'grass', [tzao] 'fry', [nru] 'meat', [tın] 'top' }\end{array}$ \\
\hline 13 & 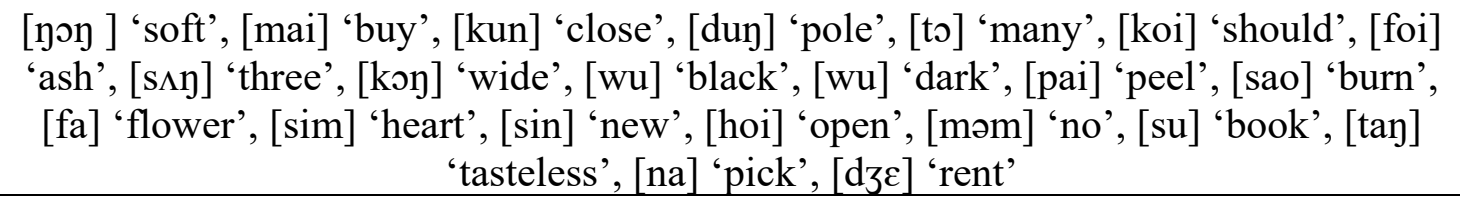 \\
\hline 31 short & $\begin{array}{c}\text { [pain] 'eight', [kio] 'foot', [bik] 'pen', [fay] 'dispatch', [dzo] 'cross', [ka] 'clip', [lak] } \\
\text { 'way', [tza] 'trace', [nam] 'threshold', [pak] 'pat', [mov] 'wood', [po] 'combine', } \\
\text { [dzok] 'chop' }\end{array}$ \\
\hline 5 & $\begin{array}{l}\text { [lain] 'spicy', [tak] 'reach', [faik] 'live', [kaik] 'hit', [waik] 'slip', [tok] 'read', [yck] } \\
\text { 'hot', [yck] 'moon', [pay] 'white', [Si] 'ten', [s b b] 'boil', [lay] 'candle', [mik] } \\
\text { 'compact', [sik] 'eat', [j o] 'drug', }\end{array}$ \\
\hline
\end{tabular}

After words has been grouped by their tone, a definition to each tone can be concluded:

Tone 15: Being defined because its beginning pitch is the lowest one can be found in Zijin Hakka while its ending pitch is the highest. 
Tone 13 and 44 is defined because tone 13 has same beginning pitch as which of tone 15 and its ending pitch is only lower than pitches of tone 44 while pitch 4 is only lower than pitch 5 .

Having define pitch 1,3,5 and 4, it is easy to define tone 11,31 and 5. But differing from others, tone 31 short means that it has same tone with 31 but its last time is relatively shorter than 31 or others tone. Tone 5 is a highest pitch can be found and of relative short length.

Thus, Zijin Hakka has a seven-tone system consisting of two rising tones /13, 15/, two flat tones $/ 11,44 /$, two falling tones $/ 31,31$ short/, and one short high tone $/ 5 / . / 31$ short/ is the shorten version of $/ 31 /$ and will be referred as $/ 31 \mathrm{~s} /$ in this paper.
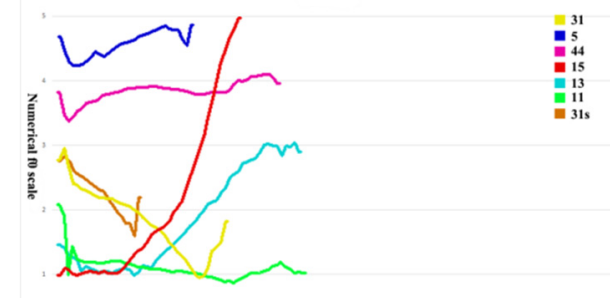

Figure 1. Seven Tones in Zijin Hakka

The Figure 1 is a collection of 7 tones in Zijin Hakka. The y axis represents the numerical $\mathrm{f} 0$ scale of pitch. All tones in figure 1 share same standard of numerical f0 scale, and their length depends on how many units in $\mathrm{x}$ axis it takes. For instance, the tone 15 (red line) in the figure begins with a pitch of numerical f0 scale 1 and ends with a pitch of numerical f0 scale 5 as well as a length of 3 units, so it is reasonable to name it tone 15 . So, tone 44 is found because it is relatively flat and has a numerical f0 scale of 4 . Tone 11 is like tone 44 as it has relatively flat tone and scale of 1 . Tone 13 begins at scale 1 and ends at scale 3, then it is reasonable to name it that way. This works to tone 31 as well. As to tone 5, it has scale of 5 but its length is apparently shorter than others, and that means that it has less syllable than others, which helps to name it tone 5 . Tone $31 \mathrm{~s}$ has similar tend with 31 but is shorter than 31 , so it is named $31 \mathrm{~s}$.

In summary, Zijin Hakka is a seven-tone dialect whose tones is consist of $31,31 \mathrm{~s}, 44,5,11,13$ and 15 .

In Zijin Hakka's seven-tone system, there are 49 (7 x 7) two adjacent syllables which is illustrated in the following Table 2:

Table 2. two adjacent syllables

\begin{tabular}{|c|c|c|c|c|c|c|c|}
\hline Syllable1/Syllable2 & 15 & 13 & 44 & 11 & 31 & $31 \mathrm{~s}$ & 5 \\
\hline 15 & $11-15$ & $11-13$ & $15-44$ & $15-11$ & $13-351$ & $13-351$ & $15-5$ \\
\hline 13 & $11-15$ & $11-13$ & $13-44$ & $13-11$ & $13-351$ & $13-351$ & $13-5$ \\
\hline 44 & $44-15$ & $44-13$ & $44-44$ & $44-11$ & $44-31$ & $44-31 \mathrm{~s}$ & $44-5$ \\
\hline 11 & $11-15$ & $11-13$ & $11-44$ & $11-11$ & $11-31$ & $11-31 \mathrm{~s}$ & $11-5$ \\
\hline 31 & $31-15$ & $31-13$ & $31-44$ & $31-11$ & $31-31$ & $31-31 \mathrm{~s}$ & $31-5$ \\
\hline $31 \mathrm{~s}$ & $31 \mathrm{~s}-15$ & $31 \mathrm{~s}-13$ & $31 \mathrm{~s}-44$ & $31 \mathrm{~s}-11$ & $31 \mathrm{~s}-31$ & $31 \mathrm{~s}-31 \mathrm{~s}$ & $31 \mathrm{~s}-5$ \\
\hline 5 & $5-15$ & $5-13$ & $5-44$ & $5-11$ & $5-31$ & $5-31 \mathrm{~s}$ & $5-5$ \\
\hline
\end{tabular}


(Note: The shaded part means that the combination keeps the lexical tone and the boldface letters show the change of the tones.)

In chart (2) eight two-adjacent syllables patterns are found to perform tonal sandhi and those patterns can group into two different rules: the rising tone sandhi rule and rising-falling tone sandhi rule. Chart (3) uses some examples to illustrate the sandhi. (4) and (5) use rule-based model to demonstrate of the rising tone sandhi rule and rising-falling sandhi rule respectively.

\subsection{Tonal Sandhi}

Table 3.Tonal combinations that undergo tone sandhi

\begin{tabular}{|c|c|c|c|}
\hline & Input & Output & Example \\
\hline \multirow[t]{4}{*}{ a. } & $13-13$ & 11-13 & {$[13 \mathrm{mai}-13 \mathrm{su}] \rightarrow[11 \mathrm{mai}-13 \mathrm{su}]$} \\
\hline & $15-13$ & 11-13 & [15bay-13fa] $\rightarrow[11 \mathrm{bay}-13 \mathrm{fa}]$ 'pull flower' \\
\hline & $15-15$ & 11-15 & {$[15 \mathrm{kai}-15 \mathrm{mao}] \rightarrow[11 \mathrm{kai}-15 \mathrm{mao}]$ 'chicken fur' } \\
\hline & $13-15$ & 11-15 & {$\left[13\right.$ hoi-15t $\left.\int \mathrm{a}\right] \rightarrow\left[11\right.$ hoi-15t $\left.\int \mathrm{a}\right]$ 'drive car } \\
\hline \multirow[t]{4}{*}{ b. } & $15-31$ & 13-351 & [15bao-31fui] $\longrightarrow[13$ bao-351fui] 'broil water' \\
\hline & $15-31 s$ & 13-351 & {$[15 \mathrm{kai}-31 \mathrm{~s} \mathrm{muy}] \rightarrow[13 \mathrm{kai}-351 \mathrm{muy}] \quad$ 'carry wood' } \\
\hline & $13-31$ & $13-351$ & [13fa-31tzao $\rightarrow[13 \mathrm{fa}-351 \mathrm{tzao}]$ 'flower grass' \\
\hline & $13-31 \mathrm{~s}$ & 13-351 & {$[13$ mai-31s bik $] \rightarrow[13$ mai-351bik $] \quad$ 'buy pen' } \\
\hline
\end{tabular}

Table 3 shows the input and output in the second and third column, and fourth column presents some example of these two adjacent syllables with IPA and their meaning. I classify the tone sandhi patterns into two categories. In the group (a) the four inputs show the adjacent rising tones sandhi, and the group (b). shows rising-falling tone sandhi.

The tonal changes shown above can be presented by the rule-based model as follow.

Rising tone sandhi rule

$$
\begin{array}{ll}
\text { i. } & 13 \rightarrow 11 /
\end{array} \text { ii. } \quad 15 \rightarrow 11 / \square\{13,15\}
$$

Rising-falling tone sandhi rule

$$
\begin{array}{ll}
\text { i. } & 15 \rightarrow 13 / \_31 \\
\text { ii. } & 15 \rightarrow 13 / \square 31 \mathrm{~s} \\
\text { iii. } & 31 \rightarrow 351 / 13 \\
\text { iv. } & 31 \mathrm{~s} \rightarrow 351 / 13
\end{array}
$$

\subsection{Bao's Model to Represent Internal Structure of Tone}

Bao (1999) proposes a model of tonal geometry to present tone feature dominated by Contour node and Register feature which is a sister of Contour; both Contour and Register are dominated by a Tonal Node. This paper will use Bao's model, that can show the internal structure of tones, to illustrate how the rising tone sandhi has assimilated alternation. 


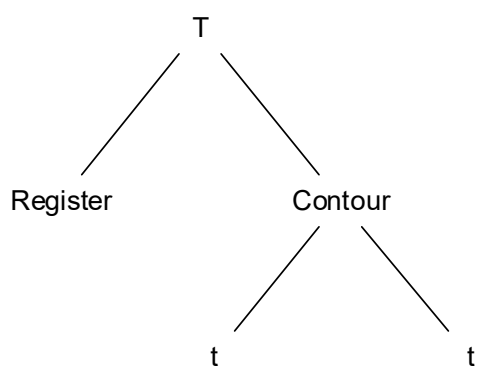

Figure 2. Bao's tonal geometry model

In Bao's model, a tone is consisting of register field and contour field. Register field could be either $\mathrm{Hr}$, marking that pitches of a tone are relatively high; or Lr, marking that pitches of a tone are relatively low. The contour field could be $l, h, l h, h l$ :

$l$ - The pitches are relatively low and maintain identical throughout the tone;

$h$ - The pitches are relatively high and maintain identical throughout the tone;

$l h$ - The pitches experience a rising process;

$h l$ - The pitches experience a falling process.

The present analysis adopts Bao's model with some differences, as illustrated below:

$\mathrm{T}$

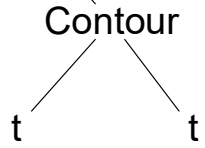

For example, the internal structure of $/ 11 /$ has a contour field of $l$, which means that $/ 11 /$ it maintains its low pitches unchanged.

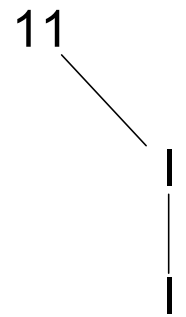

Similarly, tone 13 has a $l h$ contour field indicating that tone 13 starts with a relatively low pitch and end with a pitch higher than previous one.

13

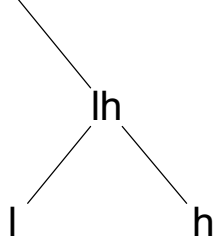

The contour structure of other tones is listed as follow:

15

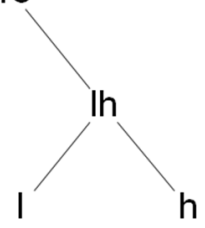

44

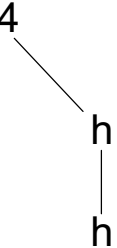

31

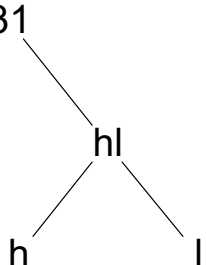

$31 s$

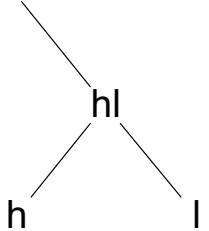

5

h

h 


\section{Rising Tone Sandhi Rule}

The (4) rising tone sandhi rule tells that any rising tone (either 13 or 15) followed by another rising tone (could be 13 or 15 tone) will change to a 11 tone in practice. Moreover, the most interesting fact is that when several rising tones cluster, they also present this rule by setting all rising tones except the last one to 11, while the last tone maintains unchanged. For instance, in Zijin Hakka, the sentence that "buy a green pepper to cook silkie soup" is expressed by a combination of seven words:
Lexical tones: (1) [mai]
(2)[tziay-ziao]
(3) [bao]
(4) [wu - kai]
(5) [ton]

$$
13
$$
1515

15
$13 \quad 15$
15
buy
green pepper
stew
black chicken
soup
'buy' 'green pepper'
'stew'
'silkie'
'soup'

Two adjacent rising tones (2) [tziay-ziao] has tone sandhi which is presented as follow:

[tziay-ziao] $\rightarrow$ [tziay-ziao]

$\begin{array}{llll}15 & 15 & 11 & 15\end{array}$

green pepper green pepper

'green pepper' 'green pepper'

When another rising tone syllable (1) [mai] is added to two adjacent rising tones (2) [tzian-ziao] which is shown above, the three adjacent rising tones [mai-tziay-ziao] will also follow the rising tone sandhi rule:

$\begin{array}{lll}\text { [mai-tzian-ziao] } & \rightarrow & \text { [mai-tzian-ziao] } \\ 13 \quad 15 \quad 15 & & 1111 \quad 15 \\ \text { buy green pepper } & \text { buy green pepper } \\ \text { 'buy green pepper' } & \text { 'buy green pepper' }\end{array}$

(4) [wu - kai] also follows the rising tone sandhi rule which is shown as follow:

$[\mathrm{wu}-\mathrm{kai}] \rightarrow$ [wu - kai]

$\begin{array}{llll}13 & 15 & 11 & 15\end{array}$

black chicken black chicken

'silkie' 'silkie'

When we combine (4) [wu - kai] and (5) [toj], the new three adjacent rising tone syllable has the same tone sandhi:

$\left.\left.\begin{array}{ccccc}{[\mathrm{wu}-\mathrm{kai}} & - & \text { toj }\end{array}\right] \rightarrow \begin{array}{ccc}{[\mathrm{wu}-\mathrm{kai}} & - & \text { toy }\end{array}\right]$

black chicken soup black chicken soup

'silkie soup' 'silkie soup'

When the rising tone (3) [bao] and [wu-kai-ton] connect to make a four adjacent rising tone combination, the tonal alternation is shown as below:

[bao - wu - kai - toy $\rightarrow$ [bao - wu - kai - toj $]$

$\begin{array}{llllllll}15 & 13 & 15 & 15 & 11 & 11 & 11 & 15\end{array}$

stew black chicken soup stew black chicken soup

'stew silkie soup' 'stew silkie soup'

When these seven rising tones cluster, they also present this rule by setting all rising tones except the last one to 11, while the last tone maintains unchanged:

Lexical tone [mai] [tziay-ziao] [bao] [wu - kai] [ton]

$13 \quad 15-15 \quad 15 \quad 13-15 \quad 15$.

buy green pepper stew black chicken soup.

'buy green pepper to stew silkie soup'

In practice, these tones will follow the rising tone sandhi rule and change to:

[mai][tziay-ziao] [bao] [wu-kai] [toy]

$11 \quad 11-11 \quad 11 \quad 11-11 \quad 15$.

buy green pepper stew black chicken soup

'buy green pepper to stew silkie soup' 
These tonal alternation shows that in Zijin Hakka, the assimilatory makes the internal structure of these combination become similar and the dissimilatory prohibits the combination of rising contour tones, which is analyzed as following.

Rising tone sandhi rule:

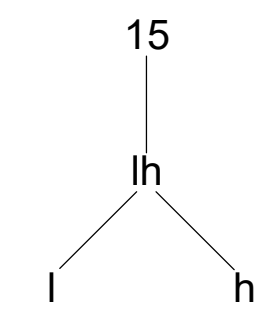

(lexical tone 15-13)

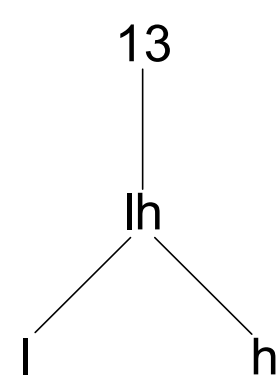

(lexical tone 13-13)
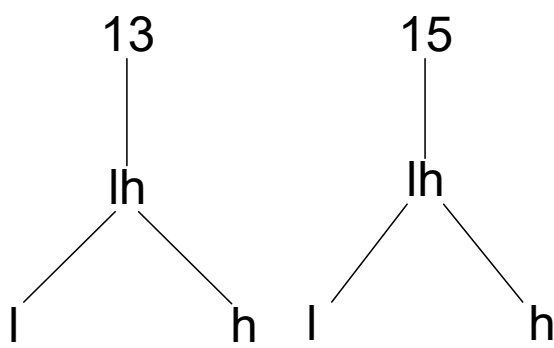

(lexical tone 13-15)
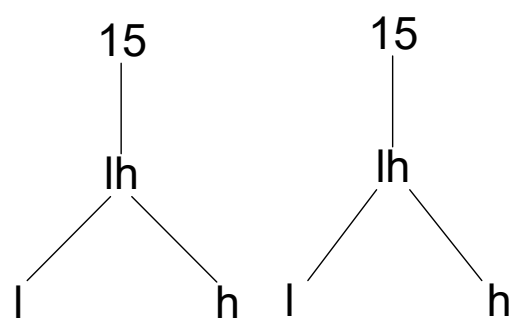

(lexical tone 15-15)
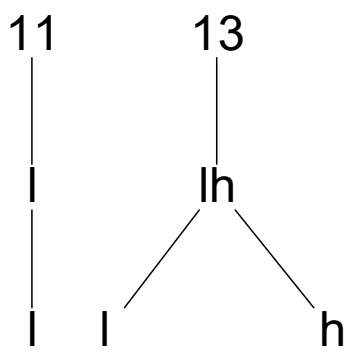

(11-13 sandhi)

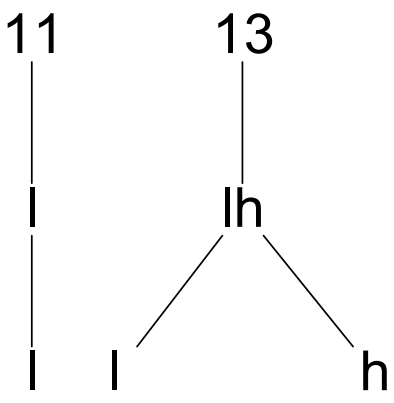

(11-13 sandhi)

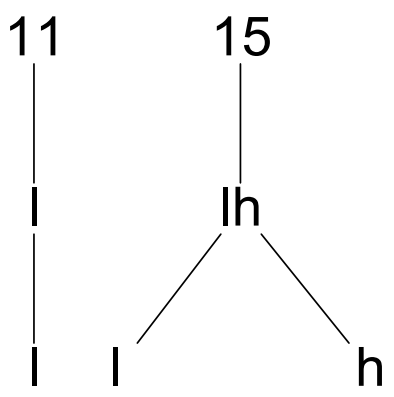

(11-15 sandhi)

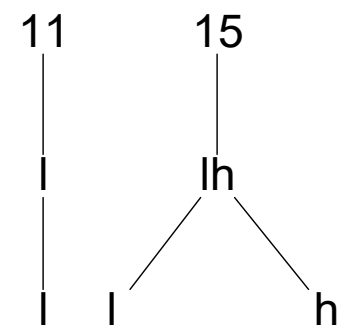

(11-15 sandhi)

In (7), assimilation happens at contour level. The lexical tone of rising tone combinations is [lh.lh] and base on the rising tone sandhi rule, it becomes $l . l h$ sandhi. This shows that the assimilatory change lh.lh to $l . l h$ making the first syllable $l h$ become $l$ which tend to become similar to the beginning tone segment of the second syllable contour $l h$.

Besides this alternation is also a dissimilated process, which can be captured by the constraint OCP-c(lh) as follow:

\subsection{The Optimality-theoretic Analysis of Rising Tone Sandhi Rule}

OCP-c(lh): Assign a * to SR who has adjacent tones of lh contour. 
OCP (Obligatory Contour Principle) is the principle for prohibiting two identical adjacent elements used by Leben (1973) and Goldsmith (1976) in research. Its definition apparently matches the principle of dissimilation. Thus, OCP is a perfect optimality-theoretic constraint to perform the effect of dissimulation. The two adjacent rising syllables 13-13, 15-13, 13-15, 15-15 prohibited base on dissimulation will be mark out by Obligatory Contour Principle at the tonal level, because both 13 and 15 are rising tone, which produce adjacent rising tones. This phenomenon can be captured by the OCP-c(lh).

As we already known that any rising tone (either 13 or 15) in Zijin Hakka followed by another rising tone (could be 13 or 15 tone) will change to a 11 tone in practice. In Lin's study, Dongshi Hakka has a completely opposite situation: Yinqu tone sandhi rule: $53 \rightarrow 55 /$ $\{31,53,32,54\}$, when falling tone 53 followed by another falling tone $31,53,32$, or 54, they will change to 55 . Lin introduce a sequential markedness constraint OCP-c(hl) to prevent adjacent hl at contour level. (Lin 2011) Although Zijin Hakka has no such kind of change between the adjacent falling tones; Lin's study is helpful that its OCP-c(hl) preventing adjacent falling tones could be modified to be OCP$\mathrm{c}(\mathrm{lh})$ as a sequential markedness constraint to avoid adjacent $l h$ at the contour level.

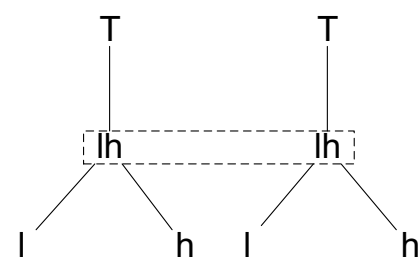

Figure 3. OCP-c(lh): Avoid adjacent lh at the contour level

F: Assign one * for every SR that is different from UR

Constraint $\mathrm{F}$ is violated by winner 11-13 which is shown as below:

Table 5. OCP-c(lh) \& F for $/ 13-15 / \rightarrow$ [11-13]

\begin{tabular}{|c|c|c|}
\hline $13-15$ & OCP-c(lh) & $\mathrm{F}$ \\
\hline $13-15$ & $* !$ & \\
\hline $11-13$ & & $* !$ \\
\hline
\end{tabular}

Another obvious tone sandhi feature in rising tone rule is that the tone sandhi always shares the left most tone feature of both syllables with the lexical tone. For example, the tone sandhi 11-15 both syllables share the left tone feature 1 with the input 13-15 at the left edge of the tone. This phenomenon can be captured by the faithfulness constraint IDENT-IO-t-L (Chen 2000). Universally, it is very common for elements at the left edge to stay intact (Beckman 1998, Nelson 1998, 2003).

\subsection{IDENT-IO-t-L}

The tone feature standing at the left edge of a tone cannot be different from its corresponding tone feature in the output. (Chen 2000).

$\mathrm{OCP}-\mathrm{c}(\mathrm{lh})$ must dominate constraint $\mathrm{F}$ to ensure the occurrence of rising tone sandhi. But the ranking of constraint $\mathrm{F}$ and constraint IDENT-IO-t-L is unknown. OCP-c(lh), when outranking constraint $\mathrm{F}$ and constraint IDENT-IO-t-L, will give priority to the rising tone sandhi. Tableau (12) (13) (14) (15) shows the effect that OCP-c(lh) dominates F and IDENT-IO-t-L. 
Table 6. OCP-c(lh) \& F \& IDENT-IO-t-L for /15-13/ $\rightarrow$ [11-13]

\begin{tabular}{|c|c|c:c|}
\hline $15-13$ & OCP-c(lh) & $\mathrm{F}$ & IDENT-IO-t-L \\
\hline $15-13$ & $* !$ & & \\
\hline $44-13$ & & $*$ & $* !$ \\
\hline $31-13$ & & $*$ & $* !$ \\
\hline $33-13$ & & $*$ & $* !$ \\
\hline
\end{tabular}

Table 7. OCP-c(lh) \& F \& IDENT-IO-t-L for $/ 13-13 / \rightarrow$ [11-13]

\begin{tabular}{|c|c|c|c|}
\hline $13-13$ & OCP-c(lh) & $\mathrm{F}$ & IDENT-IO-t-L \\
\hline $13-13$ & $* !$ & & \\
\hline $11-13$ & & $*$ & $* !$ \\
\hline $44-13$ & & $*$ & $* !$ \\
\hline $31-13$ & & $*$ & $* !$ \\
\hline $33-13$ & & $*$ & \\
\hline
\end{tabular}

Table 8. OCP-c(lh) \& F \& IDENT-IO-t-L for $/ 13-15 / \rightarrow$ [11-15]

\begin{tabular}{|c|c|c|c|}
\hline $13-15$ & OCP-c(lh) & $\mathrm{F}$ & IDENT-IO-t-L \\
\hline $13-15$ & $* !$ & & \\
\hline $11-15$ & & $*$ & $* !$ \\
\hline $31-15$ & & $*$ & $* !$ \\
\hline $33-15$ & & $*$ & $* !$ \\
\hline
\end{tabular}

Table 9. OCP-c(lh) \& F \& IDENT-IO-t-L for $/ 15-15 / \rightarrow$ [11-15]

\begin{tabular}{|c|c|c:c|}
\hline $15-15$ & OCP-c(lh) & F & IDENT-IO-t-L \\
\hline $15-15$ & $* !$ & & $* !$ \\
\hline $44-15$ & & $*$ & $* !$ \\
\hline $31-15$ & & $*$ & $* !$ \\
\hline $33-15$ & & $*$ & $*$ \\
\hline
\end{tabular}

From this analysis we can see that all the rising tone in Zijin Hakka (13 and 15) have the same tone feature standing at the left edge which is 1 . If this language has 35 tone, constraint IDENT- IO$\mathrm{t}-\mathrm{L}$ cannot determine what will happens when the input is $35+13$. Because here, the leftmost tone of the first syllable is different from the leftmost tone of the second syllable. However, I cannot find any evidence to support whether input $35+13$ will become $33+13$ or $11+13$. If we find any evidence to prove it, we need more constraints to capture this tonal alternation. But now, constraint IDENT-IO$\mathrm{t}-\mathrm{L}$ can capture all the sandhi in Zijin Hakka.

OCP-c(lh) is a crucial markedness constraint because it arranges the well-formedness of a tonal sequence rather than that of a single tone. From tableau (12) (13) (14) (15), we can see that rising 
tone sandhi has a consistent change that the first syllable changes to the 11 tone while the second syllable stay with lexical tone without any alternation. This phenomenon is captured by the constraints in the tableaus: Firstly, constraint OCP-c(lh) rules out the adjacent lh tones and then IDENT-IO-t-L prohibits the tone feature standing at the left edge of a tone from changing in the output. Naturally the winner must be the 11-13 or 11-15 in the tableaus, because there is no falling tone beginning with tone segment 1 in Zijin Hakka and if the first syllable cannot be the rising tone which is restricted by constraint OCP-c(lh), and need to maintain its tone feature standing at the left edge, which is required by constraint IDENT-IO-t-L, it has no choice but 11 tone.

Additionally, when we consider the tonal alternation of three adjacent rising tone 13-13-13 $\rightarrow 11$ 11-13 in tableau (17), we need a new constraint *peak pitch to rule out candidate 13-11-13 who does not violate any constraints of OCP-c(lh), F, and IDENT-IO-t-L.

\section{$3.3 *$ Peak Pitch}

Assign one * for every SR whose peak pitch is in first syllable.

Table 10. OCP-c(lh) \& F \& IDENT-IO-t-L\&*peak pitch for /13-13-13/ $\rightarrow$ [11-11-13]

\begin{tabular}{|c|c|c|c|c|}
\hline $13-13-13$ & OCP-c(lh) & F & IDENT-IO-t-L & *peak pitch \\
\hline $13-13-13$ & $* ! *$ & & & \\
\hline $11-11-13$ & & $*$ & & \\
\hline $11-13-13$ & $* !$ & $*$ & & \\
\hline $13-11-13$ & & $*$ & & \\
\hline $13-13-11$ & $* !$ & $*$ & & \\
\hline $13-33-33$ & & $*$ & $* !$ & \\
\hline
\end{tabular}

However, there still has a candidate 11-13-11 who does not violate any constraints of OCP-c(lh), F, IDENT-IO-t-L and * peak pitch, so we need to add another constraint to help rule out this candidate:

\section{4 *Align Last Contour Right}

The last tone contour standing at right edge of the phrase must be aligned with the corresponding tone feature in the input

Table 11. OCP-c(lh) \& F \& IDENT-IO-t-L \& *peak pitch \& Align last contour right for /13-13-13/ $\rightarrow[11-11-13]$

\begin{tabular}{|c|c|c|c|c|c|}
\hline $13-13-13$ & OCP-c(lh) & $\mathrm{F}$ & IDENT-IO-t-L & *peak pitch & Align last contour right \\
\hline $13-13-13$ & $* ! *$ & & & & \\
\hline $11-11-13$ & & $*$ & & & \\
\hline $11-13-13$ & $* !$ & $*$ & & & \\
\hline $13-11-13$ & & $*$ & & $* !$ & \\
\hline $13-13-11$ & $* !$ & $*$ & & & \\
\hline $13-33-33$ & & $*$ & $* !$ & & \\
\hline $11-13-11$ & & $*$ & & & $* !$ \\
\hline
\end{tabular}

The following tableau (20) shows the sandhi of three rising tone $15-15-15 \rightarrow 11-11-15$ 
Table 12. OCP-c(lh) \& F \& IDENT-IO-t-L \& * peak pitch \& Align last contour right for /15-15-15/

\begin{tabular}{|c|c|c|c|c|c|}
\hline \multicolumn{6}{|c|}{$\rightarrow[11-11-15]$} \\
\hline $15-15-15$ & OCP-c(lh) & $\mathrm{F}$ & IDENT-IO-t-L & *peak pitch & Align last contour right \\
\hline $15-15-15$ & $* ! *$ & & & & \\
\hline $11-11-15$ & & $*$ & & & \\
\hline $11-15-15$ & $* !$ & $*$ & & & \\
\hline $15-11-15$ & & $*$ & & $* !$ & \\
\hline $15-15-11$ & $* !$ & $*$ & & & \\
\hline $15-55-55$ & & $*$ & $* !$ & & \\
\hline $11-15-11$ & & $*$ & & & $* !$ \\
\hline
\end{tabular}

There are some tonal combinations violate the constraint *peak pitch, like 15-11, 13-44, 31-31, etc. All these tones have no sandhi, their output maintain the same as their input. In this situation, tableau (21) shows that constraint $F$ is needed to avoid changes happening in those disyllabic sequences that do not have any sandhi.

Table 13. F \& IDENT-IO-t-L \& *peak pitch \& Align last contour right for $/ 31-31 / \rightarrow[31-31]$

\begin{tabular}{|c|c|c:c:c|}
\hline $31-31$ & $\mathrm{~F}$ & $*$ peak pitch & IDENT-IO-t-L & Align last contour right \\
\hline $11-31$ & & $*$ & & \\
\hline $11-33$ & $* !$ & & $*$ & $*$ \\
\hline
\end{tabular}

As we can see from the tableau (21), candidate 11-33 violate constraint $F$ while our winner 31-31 violates constraint * peak pitch. To rule out candidate 11-33, constraint $\mathrm{F}$ must dominate constraint *peak pitch.

\section{Rising-falling Tone Sandhi Rule.}

The rising-falling tone sandhi rule has shown in the section 2 :

(4) Rising-falling tone sandhi rule:

$15 \rightarrow 13 / \_31$

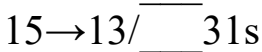

$31 \rightarrow 351 / 13$

$31 \mathrm{~s} \rightarrow 351 / 13$

This sandhi has a unique feature that both syllables of the two adjacent syllables pattern change to another tone.

(4). $\mathrm{i}$ and ii rule tells that rising tone 15 followed by a falling tone 31 or $31 \mathrm{~s}$ will change to the 13 tone, and after that when tone 31 or 31 s follow 13 tone, 31 and 31 s change to the new tone 351 , which makes me believe that 13 tone is the trigger of rule iii and iv making 31 and $31 \mathrm{~s}$ change. Besides, $31 \mathrm{~s}$ shares the same property with 31 tone in rising-falling tone sandhi. In conclusion, the rising-falling tone sandhi rule indicates that Zijin Hakka avoids the occurrence of rising tone followed by falling tone.

From the 13-31 $\rightarrow$ 13-351 alternation, $13-31 \mathrm{~s} \rightarrow 13-351$ alternation, $15-31 \rightarrow 13-351$ alternation, and $15-31 \mathrm{~s} \rightarrow 13-351$ alternation, we notice that all these four different inputs change to the same two adjacent syllables pattern which is 13-351. Besides, it can be concluded that 31 and $31 \mathrm{~s}$ is interchangeable in Zijin Hakka sandhi. Base on this phenomenon, rising-falling tone sandhi involves a neutralization process. According to Optimality theory, neutralization is the result of markedness constraints dominating the faithfulness constraints which aim at preserving variety. This process can 
be captured by the markedness constraint *1h.hl, constraint F, IDENT-IO-t-L constraint, *peak pitch, and $\mathrm{F}$ (\#lh.).

Table 14. Neutralization to $13-351$

\begin{tabular}{|c|c|}
\hline Input & Output \\
\hline $15-31$ & \\
\hline $15-31 \mathrm{~s}$ & $\mathbf{1 3 - 3 5 1}$ \\
\cline { 1 - 2 } $13-31$ & \\
\hline $13-31 \mathrm{~s}$ & \\
\hline
\end{tabular}

\section{$4.1 *$ lh.hl}

Assign one * for every SR whose first syllable is rising tone while the second syllable is falling tone.

Table 15. *lh.hl\&F for $/ 15-31 / \rightarrow[13-351]$

\begin{tabular}{|c|c|c|}
\hline $15-31$ & $*$ lh.hl & $\mathrm{F}$ \\
\hline $15-31$ & $* !$ & \\
\hline $\operatorname{13} 13-351$ & & $*$ \\
\hline
\end{tabular}

\subsection{F(\#lh.): Assign one * for Every SR Whose First Syllable is Different from that of UR When the First Syllable of UR is Rising Tone}

Constraint $* 1$ h.hl is sequential markedness constraints because it ruled out all rising-falling tone combination which is prohibited by Zijin Hakka. Besides, constraint *lh.hl must dominate the constraint $\mathrm{F}$ to prevent $\mathrm{F}$ from ruling out all possible output except lexical tone. The ranking between IDENT-IO-t-L and $\mathrm{F}$ is unknown. $\left({ }^{*} \mathrm{lh} . \mathrm{hl}>>\mathrm{F}\right.$, IDENT-IO-t-L)

Constraint $\mathrm{F}$ must dominate the constraint * peak pitch and constraint $\mathrm{F}(\# \mathrm{lh})$ to prevent output that is identical to UR from being ruled out. In other words, constraint F helps to avoid any changes happen to the two adjacent syllables sequences that do not have sandhi. Constraint F(\#lh.) must stay at low rank. The ranking between constraint * peak pitch, constraint IDENT-IO-t-L and constraint $\mathrm{F}(\# \mathrm{lh}$.) is unknown. $(* 1 \mathrm{lh} . \mathrm{hl}>>\mathrm{F}>>$ *peak pitch, IDENT-IO-t-L, F(\#lh.))

The tableau in (25) (26) (27) (28) (29) (30) shows how constraint*lh.hl, constraint F, IDENT-IO$\mathrm{t}-\mathrm{L}$ constraint, *peak pitch, and $\mathrm{F}(\# 1 \mathrm{~h}$.) functions to predict rising-falling tone sandhi.

Constraint *peak pitch helps rule out candidate 15-351 for 15-31 sandhi.

Table 16. Constraints *1h.hl \& F \& * peak pitch for 15-31

\begin{tabular}{|c|c|c|c|}
\hline $15-31$ & $*$ lh.hl & F & *peak pitch \\
\hline $15-31$ & $* !$ & & \\
\hline $15-351$ & & $*$ & \\
\hline $15-351$ & & $*$ & $* !$ \\
\hline
\end{tabular}

Constraint IDENT-IO-t-L help rule out candidate 44-351 for 15-31 sandhi. 
Table 17. Constraints *lh.hl \& F \& * peak pitch \& IDENT-IO-t-L for 15-31

\begin{tabular}{|c|c|c|c|c|}
\hline $15-31$ & $*$ lh.hl & $\mathrm{F}$ & $*$ peak pitch & IDENT-IO-t-L \\
\hline $15-31$ & $* !$ & & & \\
\hline $13-351$ & & $*$ & & $* !$ \\
\hline $44-351$ & & $*$ & & \\
\hline $15-351$ & & $*$ & $* !$ & \\
\hline
\end{tabular}

Constraint $\mathrm{F}(\# 1 \mathrm{l}$.$) help rule out candidate 11-351$

Table 18. Constraints*lh.hl \& F \& *peak pitch \& IDENT-IO-t-L \& F(\#lh.) for 15-31 sandhi

\begin{tabular}{|c|c|c|c|c|c|}
\hline $15-31$ & *lh.hl & $\mathrm{F}$ & *peak pitch & $\begin{array}{c}\text { IDENT- } \\
\text { IO-t-L }\end{array}$ & $\mathrm{F}$ (\#lh. $)$ \\
\hline $15-31$ & $* !$ & & & & \\
\hline $13-351$ & & * & & & \\
\hline $35-321$ & $* !$ & $*$ & & & \\
\hline 44-351 & & $*$ & & $* !$ & \\
\hline $15-351$ & & $*$ & $* !$ & & \\
\hline $11-351$ & & $*$ & & & $* !$ \\
\hline $15-531$ & $* !$ & $*$ & & & \\
\hline
\end{tabular}

Table 19. Constraints*lh.hl \& F \& IDENT-IO-t-L \& *peak pitch, \& F(\#lh.) for 15-31s sandhi

\begin{tabular}{|c|c|c|c|c|c|}
\hline $15-31 \mathrm{~s}$ & *lh.hl & $\mathrm{F}$ & *peak pitch & IDENT-IO-t-L & $\mathrm{F}$ (\#lh.) \\
\hline $15-31 \mathrm{~s}$ & $* !$ & & & & \\
\hline $13-351$ & & $*$ & & & \\
\hline $35-321$ & $* !$ & $*$ & & & \\
\hline $44-351$ & & $*$ & & $* !$ & \\
\hline $15-351$ & & $*$ & $* !$ & & \\
\hline $11-351$ & & $*$ & & & $* !$ \\
\hline $15-531$ & $* !$ & $*$ & & & \\
\hline
\end{tabular}

From tableau (25), (26) we can see that *lh.hl must dominate F as F rules out all the allotones except 15-31, 15-31s which is not the winner. *lh.hl rules out the allotones with lh contour in the first syllable and hl contour in the second syllable, which are candidates 15-31, 35-321 and 15-531 in tableau (25) and 15-31s, 35-321, and 15-531 in tableau(26). *peak pitch rules out candidate 15-351, IDENT-IO-t-L rules out candidate 44-451, and F(\#lh.) rules out candidate 11-351. 
Table 20. Constraints *lh.hl \& F \& *peak pitch, \& F(\#1h.) for 13-31 sandhi

\begin{tabular}{|c|c|c|c|c|}
\hline $13-31$ & $*$ lh.hl & $\mathrm{F}$ & *peak pitch & $\mathrm{F}(\#$ lh. $)$ \\
\hline $13-31$ & $* !$ & & & \\
\hline $13-351$ & & $*$ & & $*$ \\
\hline $11-321$ & & $*$ & $* !$ & $*$ \\
\hline $11-31$ & & $*$ & & $* !$ \\
\hline $11-351$ & & $*$ & & \\
\hline $13-321$ & $* !$ & $*$ & & \\
\hline
\end{tabular}

Table 21. Constraints*1h.hl \& F \& *peak pitch, \& F(\#lh.) for 13-31s sandhi

\begin{tabular}{|c|c|c|c|c|}
\hline $13-31 \mathrm{~s}$ & $* \mathrm{~h} . \mathrm{hl}$ & $\mathrm{F}$ & $*$ peak pitch & $\mathrm{F}(\# 1 \mathrm{~h})$. \\
\hline $13-31 \mathrm{~s}$ & $* !$ & & & \\
\hline $15-351$ & & $*$ & & \\
\hline $11-321$ & & $*$ & $* !$ & $* !$ \\
\hline $11-31$ & & $*$ & & $* !$ \\
\hline $11-351$ & & $*$ & & \\
\hline $13-321$ & & $*$ & & \\
\hline
\end{tabular}

In Zijin Hakka, we consider that 13-351 has a tonal rising and falling process and the rising tone extends to the tone of second syllable 351. Because of this, we believe that the last tone contour standing at right edge of the new tone 351 is aligned with the corresponding falling tone feature in the input 31, which can explain why constraint OCP-c(lh) does not make 13-351 become 33-351.

Consider rising tone sandhi and rising-falling tone sandhi together, the ranking between constraint OCP-c(lh) and constraint * $\mathrm{lh}$.hl is unknown, but must dominate constraint F; Constraint $\mathrm{F}$ must dominate constraint *peak pitch; constraint IDENT-IO-t-L, *peak pitch, *Align last contour right must dominate constraint $\mathrm{F}$ (\#lh.), which can be shown in tableau (31)

Table 22. Constraint OCP-c(lh) \& F \& IDENT-IO-t-L \& * peak pitch \& Align last contour right \& $\mathrm{F}(\#$ lh.)

\begin{tabular}{|c|c|c|c|c|c|c|}
\hline $13-13-13$ & OCP-c(lh) & $\mathrm{F}$ & *peak pitch & IDENT-IO-t-L & Align last contour right & $\mathrm{F}(\# 1 \mathrm{l})$. \\
\hline $13-13-13$ & $* ! *$ & & & & & \\
\hline $11-11-13$ & & $*$ & & & & $*$ \\
\hline $11-13-13$ & $* !$ & $*$ & & & & \\
\hline $13-11-13$ & & $*$ & $* !$ & & & \\
\hline $13-13-11$ & $* !$ & $*$ & & & & \\
\hline $13-33-33$ & & $*$ & $* !$ & $*$ & & \\
\hline $11-13-11$ & & $*$ & & & $* !$ & \\
\hline
\end{tabular}


To conclude, after this analysis, we can ascertain the current constraint ranking proposed for Zijin Hakka tone sandhi. The constraint ranking can account for rising tone sandhi and rising-falling tone sandhi. Tableau (32) shows current constraint ranking in Zijin Hakka.

\subsection{Current Constraint Ranking}

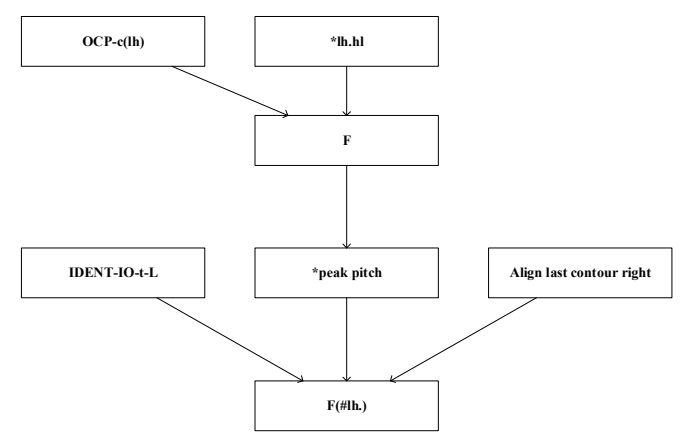

Figure 4. OCP-c(lh), *lh.hl $>>\mathrm{F}>>*$ peak pitch, IDENT-IO-t-L, Align last contour right $>>\mathrm{F}(\# \mathrm{lh}$.)

\section{Preferred Tone Before and After Rising Tone}

The constraints OCP-c(lh) and IDENT-IO-t-L indicates that, for a tone that precedes a rising tone, its first preference is $l$ or $h l$ in contour. However, since all rising tone in Zijin Hakka begins at the lowest pitch 1 [one], which means that contour beginning with pitch 1 cannot be hl in contour. In conclusion, a tone preceding a rising tone prefer $l$ in contour.

From the definition of OCP-c(lh), *lh.hl $>>\mathrm{F}>>*$ peak pitch, it is easy to conclude that a tone preceded by a rising tone prefer flat tone including tones with a contour $l$ or $h$. If otherwise (meaning that sandhi exists), these two adjacent syllables will neutralize to 13-351 for all situations.

\section{Conclusion}

Zijin Hakka's tonal sandhi are all related with rising tone and can be presented by Rising tone sandhi rule and Rising-falling tone sandhi rule. Rising tone sandhi rule is motivated by dissimilation in tone and assimilation in contour while Rising-falling tone sandhi rule performs a neutralization regarding two adjacent syllables with contour of (lh.hl). Zijin Hakka has special tone feature that all rising tones begin with lowest pitch, and it is reasonable to say that tone with contour $l$ is preferred before rising tone. As for the tone preceded by rising tone, flat tone is preferred or the whole two adjacent syllables will neutralize to 13-351.

Furthermore, in this paper, markedness and faithfulness constraints have been used to account for Zijin Hakka tone sandhi. Among these constraints, there are constraints that force adjacent element to be different (captured by OCP). OCP requires disagreement at the contour level (captured by OCP$\mathrm{c}(\mathrm{lh})$ ), which enforces the dissimilation in rising tone sandhi rule. This reflects in the fact that there are four tonal combinations that undergo tone sandhi to meet OCP requirements (i.e. 13-13, 15-13, $13-15,15-15)$. When we analyze this tone sandhi by Bao's tonal geometry model, we can find that these tonal combinations also have assimilation at the adjacent syllables contour level. Tonal sandhi of lexical tone 13-13: 11[Lr, $l]-[l h, 13] 13$. Assimilation and dissimilation are two conflict sandhi processes, but they find a balance and simultaneously exist in Rising tone sandhi rule of Zijin Hakka. Besides, neutralization plays a role in the rising-falling tone sandhi rule. According to Optimality Theory, neutralization is the result of markedness constraints dominating the faithfulness constraints which aim at preserving variety. This process can be captured by the markedness constraint *1h.hl, constraint F, IDENT-IO-t-L constraint, *peak pitch, and F(\#lh.). The markedness constraint govern the well formedness of a tonal sequence as well as of a single tone; the faithfulness constraints govern the input-output identity of elements at different levels of a tone. Four tonal combinations that undergo tone sandhi meet the requirement of these constraints (i.e.13-31, 13-31s, 15-31, 15-31s) resulting neutralization output as 13-351. Future study can have further analysis about the effects of 
expression in Zijin Hakka's tone sandhi. For example, if this language has 35 tone, what happens when the input is $35+13$ ? Because here, the leftmost tone of the first syllable is different from the leftmost tone of the second syllable. What will the output change to?

\section{Acknowledgments}

The present study is based on phonetic data collected from Yuxiang Luo, a native speaker of Zijin Hakka. Yuxiang provides accurate statistics and definitely make great contribution to this paper.

\section{References}

[1]. Bao, Zhiming. 1999. The Structure of Tone. New York \& Oxford: Oxford University Press.

[2]. Chiang, Min-Hua. 1998. Taizhongxian Dongshi Keyu Yinyun Yanjiu [Aspects of the Phonology of TungChi Hakka Language]. Taipei: National Taiwan University MA thesis. (In Chinese)

[3]. Chiang, Min-Hua. 2002. Dongshi Kejiahua de chongdie jiegou yu biandiao [Reduplication and tone-sandhi in the Tungshih Hakka dialect]. Language and Linguistics 3.3:543-567. (In Chinese)

[4]. Chiang, Jiun-Long. 2003. Liangan Dapu Kejiahua Yanjiu [The Study of Dapu Hakka in Taiwan and China]. Chiayi: National Chung Cheng University dissertation. (In Chinese)

[5]. Chen, Matthew Y. 2000. Tone Sandhi: Patterns Across Chinese Dialects. Cambridge \& New York: Cambridge University Press.

[6]. Chen, Matthew Y., Xiuhong Yan, and Lian-Hee Wee. 2004. Hakka Tone Sandhi: Corpus and Analytical Challenges. Berkeley: Project on Linguistic Analysis, University of California.

[7]. de Lacy, Paul. 1999. Tone and prominence. Manuscript. Amherst: University of Massachusetts. ROA\#333. (http://roa.rutgers.edu/).

[8]. de Lacy, Paul. 2002. The interaction of tone and stress in Optimality Theory. Phonology 19.1:132.

[9]. Lin, Hui-shan. 2011. Sequential and Tonal Markedness in Dongshi Hakka Tone Sandhi. LANGUAGE AND LINGUISTICS 12.2:313-357, 2011.

[10]. Goldsmith, John A. 1976. Autosegmental Phonology. Cambridge: MIT dissertation.

[11]. Hsiao, Yuchau E., and Yunyi Chiu. 2006. Dongshi Keyu biandiao: san zhi sizizu fenxi [Trisyllabic and tetrasyllabic tone sandhi in Dongshi Hakka]. Language and Linguistics 7.2:455482. (In Chinese)

[12]. Leben, William Ronald. 1973. Suprasegmental Phonology. Cambridge: MIT dissertation. 